\title{
Multipotent adult progenitor cells sustain function of ischemic limbs in mice
}

\author{
Xabier L. Aranguren, ${ }^{1,2}$ Jonathan D. McCue, ${ }^{3}$ Benoit Hendrickx, ${ }^{4}$ Xiao-Hong Zhu, ${ }^{5}$ Fei Du, ${ }^{5}$ \\ Eleanor Chen, 6 Beatriz Pelacho, ${ }^{2,6}$ Ivan Peñuelas,7 Gloria Abizanda, ${ }^{2}$ Maialen Uriz, ${ }^{2}$ \\ Sarah A. Frommer, ${ }^{6}$ Jeffrey J. Ross, ${ }^{6}$ Betsy A. Schroeder, ${ }^{6}$ Meredith S. Seaborn, ${ }^{6}$ \\ Joshua R. Adney, ${ }^{6}$ Julianna Hagenbrock, ${ }^{6}$ Nathan H. Harris, ${ }^{6}$ Yi Zhang, ${ }^{5}$ Xiaoliang Zhang, ${ }^{5}$ \\ Molly H. Nelson-Holte, ${ }^{6,8}$ Yuehua Jiang, ${ }^{6}$ An D. Billiau, ${ }^{8,9}$ Wei Chen, ${ }^{5}$ Felipe Prósper, ${ }^{2}$ \\ Catherine M. Verfaillie, 6,8 and Aernout Luttun ${ }^{1,6}$
}

\begin{abstract}
${ }^{1}$ Center for Molecular and Vascular Biology, Katholieke Universiteit Leuven, Leuven, Belgium. 2Hematology and Cell Therapy Area, Clínica Universitaria and Foundation for Applied Medical Research, University of Navarra, Pamplona, Spain. ${ }^{3}$ Department of Surgery, University of Minnesota, Minneapolis, Minnesota, USA. ${ }^{4}$ Department of Plastic, Reconstructive and Aesthetic Surgery, Katholieke Universiteit Leuven, Leuven, Belgium. ${ }^{5}$ Department of Radiology, Center for Magnetic Resonance Research, and ${ }^{6}$ Department of Medicine, Stem Cell Institute, University of Minnesota, Minneapolis, Minnesota, USA. 'Department of Nuclear Medicine, Clínica Universitaria and MicroPET Research Unit CIMA-CUN, University of Navarra, Pamplona, Spain. ${ }^{8}$ Stem Cell Institute Leuven and ${ }^{2}$ Laboratory of Experimental Transplantation, Katholieke Universiteit Leuven, Leuven, Belgium.
\end{abstract}

\begin{abstract}
Despite progress in cardiovascular research, a cure for peripheral vascular disease has not been found. We compared the vascularization and tissue regeneration potential of murine and human undifferentiated multipotent adult progenitor cells (mMAPC-U and hMAPC-U), murine MAPC-derived vascular progenitors (mMAPC-VP), and unselected murine BM cells (mBMCs) in mice with moderate limb ischemia, reminiscent of intermittent claudication in human patients. mMAPC- $U$ durably restored blood flow and muscle function and stimulated muscle regeneration, by direct and trophic contribution to vascular and skeletal muscle growth. This was in contrast to mBMCs and mMAPC-VP, which did not affect muscle regeneration and provided only limited and transient improvement. Moreover, mBMCs participated in a sustained inflammatory response in the lower limb, associated with progressive deterioration in muscle function. Importantly, mMAPC-U and hMAPC-U also remedied vascular and muscular deficiency in severe limb ischemia, representative of critical limb ischemia in humans. Thus, unlike BMCs or vascular-committed progenitors, undifferentiated multipotent adult progenitor cells offer the potential to durably repair ischemic damage in peripheral vascular disease patients.
\end{abstract}

\section{Introduction}

Peripheral vascular disease (PVD) is a growing health problem in Western societies. PVD presents itself with different degrees of severity, e.g., intermittent claudication (IC) is an early moderate manifestation and critical limb ischemia (CLI), a more chronic and severe problem typically involving tissue loss. Bypass surgery or balloon dilatation offer benefit, but many patients with moderate-to-severe PVD cannot be helped by such interventions due to the presence of life-threatening comorbidities or the diffuse nature of the underlying vascular disease (1). Furthermore, many CLI patients do not respond to current treatments and require limb amputation (2). For these "no-option" patients, 2 noninvasive, alternative revascularization strategies are being tested in the clinic: single angiogenic gene/protein and cell-based therapies. Unfortunately, the outcome of clinical trials using gene/protein-based therapy has been disappointing (3, 4). The failure of this approach is likely due to several factors, including an incomplete molecular understanding of the complex vascular growth process, the likely necessity for concurrent use of multiple angiogenic factors, and instability of currently

Nonstandard abbreviations used: ADSC, adipose tissue-derived stem cell; $\mathrm{BMC}, \mathrm{BM}$ cell; CLI, critical limb ischemia; EPC, endothelial progenitor cell;

$\mathrm{h}$, human; IC, intermittent claudication; MAPC, multipotent adult progenitor cell; $\mathrm{m}$, murine; MAPC-U, undifferentiated MAPCs; MAPC-VP, MAPC-derived vascular progenitors; MSC, mesenchymal stem cell; PVD, peripheral vascular disease;

SkMB, skeletal myoblast.

Conflict of interest: Catherine M. Verfaillie is a consultant with Athersys Inc.

Citation for this article: J. Clin. Invest. 118:505-514 (2008). doi:10.1172/JCI31153. used factors to achieve long-term benefits (5). Additionally, the dysfunctional endogenous vascular cells may fail to respond to these factors, and thus, administration of functional vascular cells may be needed. While transplantation with (vascular) stem cells may offer functional revascularization and, therefore, protection against tissue loss in patients with mild or severe forms of PVD, this therapy does not replace already-lost tissue. This has led to the hypothesis that treatment with multipotent stem cells - able to generate blood vessels as well as replace lost target tissue - may provide significantly more benefit for patients suffering from more severe forms of PVD, e.g. CLI.

Several cell types, including BM cells (BMCs), ES cells, mesenchymal stem cells (MSCs), skeletal myoblasts (SkMBs), umbilical cord or peripheral blood cells, adipose tissue-derived stem cells (ADSCs), and endothelial progenitor cells (EPCs), have been tested for their effect on restoring blood supply and/or muscle function in ischemic limbs (6-13). Despite encouraging results from preclinical and small uncontrolled clinical trials $(14,15)$, many questions concerning stem cell-based therapy remain to be answered prior to its widespread use. For example, the long-term efficacy and safety of this approach (e.g., some grafts may contain cells that are harmful to already-compromised tissue) has not been fully evaluated. It has not yet been determined whether cells that also can regenerate the ischemic tissue aside from vascular restoration are superior to cells able to aid only in revascularization and whether these cells are effective in both moderate and more severe forms of PVD. There is also little information as to the importance of the differentiation status of the transplanted cells (16), i.e., Will 



Figure 1

mMAPC-U and hMAPC-U engraftment and differentiation. (A-F) At 14 days, cell patches (dotted lines in $\mathbf{A}$ and $\mathbf{B})$ were found by in vivo (A) or ex vivo (B) imaging, GFP fluorescence (C), and anti-GFP staining in cross sections (D). Note positioning of the patches near $\alpha$-SMA ${ }^{+}$(red in E) vessels and the surrounding CD45+ (red in F) clusters. (G-I) At 14 days, confocal imaging revealed some GFP+ cells coexpressing CD31 or $\alpha$-SMA (arrowheads in $\mathbf{G}$ and $\mathbf{H}$, respectively). A limited number of regenerating SkMBs were GFP+ (thus donor derived; revealed by anti-GFP staining; I). (J-L) At 5 weeks, mMAPC-U persisted (shown by in vivo imaging, J; and anti-GFP staining, $\mathbf{K}$ and $\mathbf{L}$ ). Note that donor-derived regenerating fibers (GFP+ cells in $\mathbf{K}$ ) were still apparent and that cell number was less than it was at 14 days (compare $\mathbf{D}$ and $\mathbf{L})$. (M-P) At 30 days, hMAPC-U stably engrafted (revealed by large human vimentin+ [green] cell patches intercalated between muscle fibers [M and $\mathbf{N}$ ] and as scattered cells around vessels [P]). Cells were detected in the endothelial layer of vessels by a human-specific anti-CD31 antibody (green in O), indicating EC differentiation, and in some cells the human vimentin signal (green) colocalized (yellow; white arrowheads) with $\alpha$-SMA (red), indicating their SMC identity in P. Images in A, C, D, E, G-J, L, M, O, and $\mathbf{P}$ are from adductor; images in B, F, I, and $\mathbf{K}$ from gastrocnemius and $\mathbf{N}$ from quadriceps muscle. DAPI was used as nuclear counterstain in $\mathbf{C}$ and $\mathbf{E}$ and $\mathbf{M}-\mathbf{O}$ and Topro (Molecular Probes) in $\mathbf{H}$ and $\mathbf{P}$. Scale bars: $50 \mu \mathrm{m}(\mathbf{G}-\mathbf{I}, \mathbf{K}), 100 \mu \mathrm{m}$ (C, D, F, L, M, O, and P), $200 \mu \mathrm{m}$ (E and N), and $500 \mu \mathrm{m}$ (A, B, and J).

better results be achieved with fully mature cells, with progenitors committed to a certain cell type, or with immature noncommitted stem cells that have the capacity to differentiate into vascular and nonvascular tissue cells? For instance, preclinical studies have suggested that in contrast to EPCs, terminally differentiated ECs do not improve revascularization $(8,17)$. The challenge of addressing these issues is further increased by the likelihood that results from studies with animal cells may not necessarily be extrapolated to those with human cells.

Here, we first compared the benefits of 2 cell populations with the ability to generate vascular cells and SkMBs and 1 population of vascular predifferentiated cells in restoring perfusion and mus- cle function in a moderate mouse limb ischemia model, reflecting the clinical status of IC patients. The first population with vascular and muscle differentiation ability were unmanipulated murine BMCs (mBMCs), containing a mixture of stem cells capable of vascular and/or muscular differentiation (18-21). The second population was murine undifferentiated multipotent adult progenitor cells (mMAPC-U), which can - at the single-cell level - differentiate into multiple cell types from any of the 3 germ layers of an embryo $(22,23)$. We also grafted murine MAPC-derived vascular progenitors (mMAPC-VP) containing endothelial and smooth muscle-committed progenitors, which can further differentiate in mature ECs and smooth muscle cells (SMCs) but not SkMBs. 




Figure 2

mMAPC-VP and mBMC engraftment and differentiation. (A-C) At 14 days, mMAPC-VP were lower in number compared with mMAPC-U (compare $\mathbf{A}$ and Figure $1 \mathrm{C}$ ). Confocal imaging revealing more GFP+ cells coexpressing CD31 (B) and $\alpha$-SMA (arrowheads in C). Overexposure in the DAPI channel was used to reveal muscle tissue in A. (D-G) At 14 days, anti-GFP staining revealed that mBMCs engrafted mostly in connective and fat tissue outside muscle (D) and only occasionally intercalated between muscle cells (E). Confocal analysis showing that mBMCs (green; F) did not integrate in but were located outside (arrowheads in F) vessels (CD31; red; $\mathbf{F}$ ) and that most of the cells (green; G) colocalized (yellow; G) with the pan-hematopoietic marker CD45 (red; G). Images in $\mathbf{A}-\mathbf{D}, \mathbf{F}$, and $\mathbf{G}$ are from adductor; the image in $\mathbf{E}$ is from gastrocnemius muscle. Topro was used as nuclear counterstain in F. Scale bars: $40 \mu \mathrm{m}$ (B and C), $62.5 \mu \mathrm{m}$ (F and G), $100 \mu \mathrm{m}$ (D and E), and $150 \mu \mathrm{m}$ (A).

In a second phase, we tested the effect of mMAPC-U and human MAPC-U (hMAPC-U) in a severe murine limb ischemia model, reflecting the clinical status of CLI patients.

\section{Results}

Cell engraftment and differentiation. We injected $1 \times 10^{6} \mathrm{mMAPC}-\mathrm{U}$, mMAPC-VP, or mBMCs in the left adductor and gastrocnemius region after femoral artery ligation in $\mathrm{C} 57 \mathrm{BL} / 6$ mice or $1 \times 10^{6}$ hMAPC-U into the left adductor and gastrocnemius region of $\mathrm{BALB} / \mathrm{c}-n u / n u$ mice after iliac artery ligation/transsection (Supplemental Figure 1; supplemental material available online with this article; doi:10.1172/JCI31153DS1). Fourteen days after transplantation, $\mathrm{GFP}^{+}$mMAPC-U engrafted adductor and gastrocnemius muscles in large patches $(20-800$ cells in size on a single cross section), as revealed by direct fluorescence (Figure 1, A-C, and Supplemental Figure 1) or anti-GFP immunohistochemistry (Figure 1D). Cells intercalated between muscle fibers in the vicinity of $\alpha-\mathrm{SMA}^{+}$vessels and were surrounded by $\mathrm{CD} 45^{+}$cells (Figure $1, \mathrm{E}$ and $\mathrm{F})$. Confocal analysis revealed that mMAPC-U differentiated into $\mathrm{CD}^{+} 1^{+} \mathrm{ECs}$ and $\alpha$-SMA ${ }^{+} \mathrm{SMCs}$ (Figure 1, G and H); however, only $2.8 \% \pm 1.0 \%$ and $2.6 \% \pm 0.6 \%$ of the retained $\mathrm{GFP}^{+}$cells adopted an EC or SMC phenotype, respectively. Anti-GFP staining and direct fluorescence revealed that MMAPC-U contributed to (regenerating) muscle fibers in the damaged gastrocnemius, which was at least in part due to fusion with host muscle cells (Figure 1 I and Supplemental Figure 2). Most cells remained in an undifferentiated state, given their persistent CD117 expression (data not shown). At 5 weeks after transplantation, $\mathrm{GFP}^{+}$cells were still detected, and their direct contribution to regenerating muscle was still apparent (Figure 1, J-L). However, the total number of $\mathrm{GFP}^{+}$ cells had decreased significantly (patches contained only 20-120 cells; no. of cells $/ \mathrm{mm}^{2}: 7 \pm 1$ at 5 weeks versus $20 \pm 6$ at 14 days; $P=0.07$; Figure $1 \mathrm{~L}$ ). No contralateral engraftment or circulating $\mathrm{GFP}^{+}$cells were detected (Supplemental Figure 1). In no instance did we see tumor formation. hMAPC-U robustly engrafted adductor muscles, revealed by large clusters of human-specific vimentin ${ }^{+}$cells $\left(9 \pm 3\right.$ cells $/ \mathrm{mm}^{2}$ muscle tissue at day 30; Figure 1, M and N), intercalated between muscle fibers. hMAPC-U differentiated into ECs that stained with human-specific anti-CD31 antibodies $(8.2 \% \pm 1.2 \%$; Figure 10$)$ and - to a lesser extent - into $\alpha$-SMA ${ }^{+}$SMCs $(1.3 \% \pm 0.6 \%$, revealed by colocalization of human-specific vimentin and $\alpha$-SMA; Figure $1 \mathrm{P})$.

mMAPC-VP (an equal mix of EC and SMC precursors predifferentiated for 9 and 6 days in VEGF-A or TGF- $\beta 1$, respectively; for their characterization, see Supplemental Figure 3 and ref. 24, respectively) had a different engraftment pattern. $\mathrm{GFP}^{+}$cells were more scattered and fewer in number (no. of cells $/ \mathrm{mm}^{2}: 8 \pm 2$ for mMAPC-VP versus $20 \pm 6$ for mMAPC-U; $P<0.05$; Figure $2 \mathrm{~A}$ ). Compared with mMAPC-U, 3- to 4-fold more retained $\mathrm{GFP}^{+}$cells coexpressed vascular markers (CD31: 9.6\% $\pm 2.7 \%$, Figure $2 \mathrm{~B}$; $\alpha$-SMA: $9.5 \% \pm 5.3 \%$, Figure $2 \mathrm{C}$ ). mMAPC-VP did not contribute to SkMBs. Like mMAPC-U, mMAPC-VP were surrounded by $\mathrm{CD}_{4} 5^{+}$cells (data not shown). mBMCs lodged in connective tissue and fat pads outside muscle bundles and in skin, and only a few were found intercalated between muscle fibers (Figure 2, D and E). The number of mBMCs at 14 days was 3 -fold less than mMAPC-U (no. of cells $/ \mathrm{mm}^{2}: 7 \pm 1$ for $\mathrm{mBMCs}$ versus $20 \pm 6$ for mMAPC-U; $P<0.05$ ). No direct contribution of mBMCs to vessels or SkMBs was seen; instead, cells resided outside vessels, and the majority $(97.9 \% \pm 2.6 \%)$ costained with the pan-hematopoietic marker CD45 (Figure 2, F and G).

Early effects of $m M A P C-U, m M A P C-V P$, and $m B M C$ s in moderate limb ischemia. First, we compared the potential of mMAPC-U, mMAPC-VP, and $\mathrm{mBMCs}$ injected on the left side, immediately after femoral artery ligation in C57BL/6 mice, a model of moderate limb ischemia, reminiscent of IC in humans (25). Both sides were ligated (except in laser Doppler studies, where only the left side was ligated), which allowed evaluation of a possible contralateral effect of the graft. Nine days after transplantation, there was a 3-fold increase in $\alpha-\mathrm{SMA}^{+}$area and a 1.4-fold increase in capillary density in the adductor of mMAPC-U-treated animals compared with vehicle-treated control animals (Figure 3, A and B, and Table $1)$. The $\alpha-\mathrm{SMA}^{+}$area in the adductor of $\mathrm{mBMC}$ - and mMAPCVP-treated animals was increased over vehicle control, but significantly less than in mMAPC-U-treated animals, while capillary density was similarly increased in all cell-treated groups (Figure 3, $\mathrm{A}-\mathrm{D}$, and Table 1). Flow recovery in the limb of mMAPC-U-treated animals was significantly better compared with that in vehicletreated mice, and intermediate in MBMC- and mMAPC-VP-treated animals (Figure 3E and Table 1). No vascular expansion was seen in the contralateral adductors of cell-treated animals (data not shown). To evaluate limb function, we used a swim endurance test (26). Consistent with improved limb perfusion, swim performance of mMAPC-U-treated animals was significantly better than that of vehicle-injected mice - approaching the swim capacity of mice in which only 1 femoral artery was ligated and that did not receive cell therapy - while $\mathrm{mBMC}$ - or mMAPC-VP-treated animals performed at an intermediate level (Figure $3 \mathrm{E}$ and Table 1). 



Figure 3

Early effects of mMAPC-U, mMAPC-VP, and mBMCs in moderate ischemia. (A-D) $\alpha-S M A$ (red) staining at 9 days in adductor of vehicle- (A), mMAPC-U- (B), mBMC- (C), or mMAPC-VP-treated mice (D). Overexposure in the DAPI channel was used to reveal muscle tissue. (E) Left: Laser Doppler measurements in left legs (expressed as \% versus the nonligated right leg) before $(-)$ and after (+) ligation of vehicle- (red), mMAPC-U(black), mBMC- (gray), or mMAPC-VP-treated mice (blue). Right: Swim endurance test measuring hind limb function (expressed as \% of baseline [day -1] performance) in vehicle- (red), mMAPC-U- (black), mBMC(gray), or mMAPC-VP-injected mice (blue). The dotted line indicates the performance level of mice in which only one femoral artery was ligated 9 days prior and that were untreated. $(\mathbf{F}-\mathbf{H})$ Magnetic resonance spectra (MRS) recorded at 9 days to determine the energetic status (expressed as $\Sigma\left[\mathrm{PCr} / \mathrm{P}_{\mathrm{i}}, \mathrm{PCr} / \gamma-\mathrm{ATP}\right]$, with 23 as normal reference value) in the lower limb muscle showing representative overlays of the spectrum of a nonischemic mouse (gray in $\mathbf{F}-\mathbf{H}$ ) and those of vehicle(red; F), mMAPC-U- (blue; G), and mBMC- or mMAPCVP-injected mice (green; $\mathbf{H}$ ). ${ }^{*} P<0.05$ versus vehicle for each corresponding time point. Scale bars: $100 \mu \mathrm{m}$.
In support of a better functional status, mMAPC-U-transplanted animals lifted their limbs more vigorously compared with vehicleinjected animals (Supplemental Video 1). Improved muscle function correlated with better muscle energetics, as shown by magnetic resonance spectroscopy/imaging (MRSI), measured in the most severely affected calf region (Supplemental Figure 4). The sum of the $\mathrm{PCr} / \mathrm{P}_{\mathrm{i}}$ (phosphocreatine/inorganic phosphate) and $\mathrm{PCr} / \gamma$-ATP ratios - which are both lowered in the case of impaired energetics (Supplemental Figure 4) - was significantly higher in mMAPC$\mathrm{U}$-treated than in vehicle-injected calf muscles, while an intermediate but variable and statistically nonsignificant increase was seen in $\mathrm{MBMC}$ - and mMAPC-VP-treated animals (Figure 3, F-H, and Table 1).

Late effects of $m M A P C-U, m M A P C-V P$, and $m B M C$ s in moderate limb ischemia. We reevaluated the mice between 9 and 35 days after ligation. In vehicle control animals, a spontaneous recovery to $52 \%$ perfusion was seen 3 weeks after transplantation, while flow recovered progressively to $83 \%$ in mMAPC-U-treated animals (Figure $4 \mathrm{~A}$ and Table 1 ). In contrast, lower limb perfusion of mMAPC-VPtreated animals reached a plateau by day 9, while mBMC-treated animals deteriorated between days 12 and 21 (from $53 \%$ to $47 \%$ ), so that flow was no longer significantly different from that in control animals (Figure
4A and Table 1). By day 21, swim endurance recovered in vehicle control mice to $45 \%$ of baseline, while it became nearly normal ( $85 \%$ of baseline) in mMAPC-U-treated animals (Figure 4B and Table 1). However, swim endurance reached a plateau by day 14 in mMAPC-VP-treated animals, while it progressively decreased in mBMC-treated animals (from $49 \%$ to $14 \%$; Figure $4 \mathrm{~B}$ and Table 1). Histological evaluation of the gastrocnemius muscle at 4 weeks demonstrated significant differences between treat-
Table 1

Early and late effects of mMAPC-U, mMAPC-VP, and mBMCs on limb perfusion, status, and function in moderate limb ischemia

Vehicle mMAPC-U mBMCs mMAPC-VP

\section{Early phase (day 9)}

Vascular bed (adductor)

$\begin{array}{lcccc}\text { Collaterals }\left(\% \alpha-S M A^{+} \text {area); } n=9\right. & 0.9 \pm 0.1 & 2.7 \pm 0.3^{\mathrm{A}, \mathrm{B}} & 1.6 \pm 0.3^{\mathrm{A}} & 1.3 \pm 0.2^{\mathrm{C}} \\ \text { Capillaries }\left(\mathrm{no} . / \mathrm{mm}^{2}\right) ; n=9 & 580 \pm 48 & 807 \pm 46^{\mathrm{A}} & 734 \pm 65^{\mathrm{A}} & 868 \pm 93^{\mathrm{A}} \\ \text { d flow }(\%) ; \mathrm{D} n=6-8 & 39 \pm 2 & 63 \pm 9 \mathrm{~A}, \mathrm{~B} & 48 \pm 5^{\mathrm{A}} & 47 \pm 2^{\mathrm{A}} \\ \text { m endurance }(\%) ; \mathrm{E} n=14-7 & 23 \pm 6 & 58 \pm 12^{\mathrm{A}} & 49 \pm 8^{\mathrm{A}} & 45 \pm 8^{\mathrm{A}} \\ \text { SI (AU); } ; n=4-6 & 12 \pm 2 & 18 \pm 2^{\mathrm{A}} & 16 \pm 3 & 16 \pm 2\end{array}$

\section{Late phase (days 21-35)}

Blood flow (\%); day $21 ; n=5-6$ Swim endurance $(\%) ; \mathrm{E}$ day $21 ; n=6$

Tissue viability $(\%) ; \mathrm{G}$ day $28 ; n=5$

Fibrosis $(\%) ;$ Hay $28 ; n=5$

Toe necrosis (\%); day $35 ; n=15-17$

$\begin{array}{ccc}83 \pm 1^{A, B} & 47 \pm 7 & 50 \pm 1 \\ 85 \pm 13^{B, C} & 14 \pm 4^{A} & 44 \pm 9 \\ 74 \pm 5^{A, B} & 23 \pm 6^{A} & 43 \pm 4 \\ 6 \pm 1^{A, B} & 14 \pm 2^{A} & 10 \pm 1 \\ 0 & 69 & 30\end{array}$

Data represent the mean \pm SEM). ${ }^{A} P<0.05$ versus vehicle; ${ }^{B} P<0.05$ versus $m B M C$ and $m M A P C-V P$; ${ }^{C P}=0.06$ versus vehicle. ${ }^{D} \%$ of nonligated leg; $E \%$ of baseline endurance; ${ }^{F}$ sum of $P C r / P_{i}$ (phosphocreatine/inorganic phosphate) and PCr/ $\gamma$-ATP ratios; G\% TTC ${ }^{+}$area of total muscle area; ${ }^{\circ} \%$ Sirius red ${ }^{+}$ area of total muscle area; 'incidence. MRSI, magnetic resonance spectroscopy/imaging; TTC, tetraphenyltetrazolium chloride. 



\begin{tabular}{|c|c|c|c|}
\hline C & $\begin{array}{c}\text { clini } \\
\text { toe } \\
\text { necrosis }\end{array} \mid$ & $\begin{array}{l}\text { ical sta } \\
\left|\begin{array}{c}\text { sudden } \\
\text { death }\end{array}\right|\end{array}$ & \\
\hline vehicle & $5 / 15$ & $0 / 15$ & \\
\hline MMAPC-U & $0 / 15$ & $0 / 15$ & \\
\hline$m B M C$ & $11 / 16$ & $2 / 16$ & \\
\hline$m M A P C-V P$ & $5 / 17$ & $0 / 17$ & \\
\hline
\end{tabular}




vehicle



$m M A P C-U$



$m B M C$



$m M A P C-V P$
Figure 4 surements in left legs (expressed as \% of the nonligated right leg) of vehicle- (red), mMAPC-U(black), mBMC- (gray), or mMAPC-VP-treated mice (blue). (B) Swim performance (expressed as \% versus day -1 [baseline]) of vehicle- (red), mMAPC-U- (black), mBMC- (gray), or mMAPC-VP-treated mice (blue). (C) Table summarizing clinical evaluation of all treatment regimens up to 5 weeks after transplantation. Representative images (D-G) and corresponding quantification $(\mathbf{H})$ of whole-tissue (gastrocnemius muscle, cut into 2 slices of equal thickness) viability (red, viable tissue) by triphenyltetrazolium chloride (TTC) staining 4 weeks after transplantation in vehicle (D; red in H), mMAPC-U (E; black in $\mathbf{H})$, mBMC (F; gray in $\mathbf{H})$, and mMAPC-VP (G; blue in $\mathbf{H})$ groups. (I-L) Sirius red-stained cross sections of gastrocnemius muscle 5 weeks after transplantation of vehicle- (I), mMAPC-U- (J), mBMC(K), or mMAPC-VP-injected (L) animals. All analyses were performed on 6-12 mice per group. The insets in $\mathbf{E}$ and $\mathbf{J}$ show a nonischemic muscle. ${ }^{*} P<0.05$ versus vehicle for each corresponding time point. Scale bars: $500 \mu \mathrm{m}(\mathbf{I}-\mathbf{L})$. Original magnification, $\times 2(\mathbf{D}-\mathbf{G}) ; \times 0.66$ (inset E); $\times 2.5$ (inset J).
Late effects of mMAPC-U, mMAPC-VP, and mBMCs in moderate ischemia. (A) Laser Doppler mea-

a modest level of fibrosis, compared with that from vehicle control animals (Figure 5, B, C, and F). In contrast, in mMAPC-VP- and mBMCtreated animals, the level of fibrosis was quite variable but overall significantly higher than in vehicle-treated control animals (Figure 5, B and D-F). Compared with other groups, the inflammatory infiltrate of the gastrocnemius muscle was markedly increased in the $\mathrm{mBMC}$ group (Figure $5, \mathrm{G}-\mathrm{K})$ consisting of host and donor $\left(\mathrm{GFP}^{+} \mathrm{CD} 45^{+}\right)$cells, mostly CD $11 \mathrm{c}^{+}$dendritic cells and $\mathrm{CD}^{+} \mathrm{T}$ lymphocytes (Supplemental Figure 5 ). Muscle regeneration (defined by the presence of fibers with central nuclei) in mMAPC-U-treated animals was much more pronounced than in the other treatment groups (Figure 5L). This was confirmed by desmin staining, which is known to be strongly positive in small regenerating myogenic cells (27) (Figure 5, M-Q). Moreover, 3-fold more $\mathrm{N}-\mathrm{CAM}^{+}$cells (a satellite cell marker; ref. 28) were present in regenerating areas of the gastrocnemius of mMAPC-U-treated animals than in other groups (Figure 5, R-V). No beneficial effects on fibrosis, necrosis, or regeneration were seen in the contralateral muscle of mMAPC-Utreated animals (data not shown).

Trophic effects of $m M A P C-U$ on vascular and muscle cells. We hypothesized that the improvement in muscle perfusion, function, and regeneration in mMAPC-U-grafted animals ment groups. While mMAPC-U-treated animals did not show any overt necrotic (Figure 4, E and $\mathrm{H}$, and Table 1) or fibrotic (Figure 4J) regions, small areas of necrosis (Figure 4, D, G, and $\mathrm{H}$, and Table 1) and fibrosis (Figure $4, \mathrm{I}$ and $\mathrm{L}$, and Table 1) remained in vehicle control and mMAPC-VP-treated muscles. By contrast, the gastrocnemius of more than $50 \%$ of $\mathrm{mBMC}$ treated animals showed severe signs of necrosis (Figure 4, F and $\mathrm{H}$, and Table 1) and fibrosis (Figure $4 \mathrm{~K}$ and Table 1 ). In addition, clinical observation revealed no cases of toe/limb necrosis in mMAPC-U-treated animals, while this was observed in 33\%, $30 \%$, and $69 \%$ of vehicle control, mMAPC-VP-, and mBMC-treated mice, respectively (Figure $4 \mathrm{C}$ and Table 1).

Differential effects of $m M A P C-U, m M A P C-V P$, and $m B M C$ in moderate limb ischemia. Since muscle function reached a plateau in mMAPC-VP-injected mice and started to deteriorate in $\mathrm{mBMC}$ treated mice around day 14, we performed histological analyses on gastrocnemius muscle at this "hinge" point. Compared with vehicle-treated animals, mMAPC-U- and mMAPC-VP-, but not mBMC-treated, animals had significantly less muscle necrosis (Figure 5A). Muscle from mMAPC-U-treated mice demonstrated was in part the result of trophic factor production (8). Indeed, we found that, in vitro, mMAPC-U secreted substantial amounts of VEGF-A, PDGF-BB, IGF-1 (417 $\pm 8,33 \pm 3$, and $198 \pm 20 \mathrm{pg} / 10^{5}$ cells/60 h, respectively), factors important for EC, SMC, and/or SkMB proliferation, and monocyte chemoattractant protein-1 (MCP-1) $\left(25 \pm 3 \mathrm{pg} / 10^{5}\right.$ cells $\left./ 60 \mathrm{~h}\right)$, important for initiating collateral expansion (29). VEGF-A and IGF-1 protein could be detected in areas surrounding grafted cells in vivo $(41 \% \pm 2 \%$ of the mMAPC-U stained positive for VEGF; IGF-1 could be detected mainly in the vicinity of engrafted cells; Supplemental Figure 6). Compared with mMAPC-U, mBMCs produced significantly less VEGF-A and IGF-1 $(P<0.05)$ in vitro, and similar levels of MCP- 1 and PDGF-BB $\left(60 \pm 6,79 \pm 2,25 \pm 2\right.$, and $43 \pm 9 \mathrm{pg} / 10^{5}$ cells $/ 60 \mathrm{~h}$, respectively). mMAPC-U-conditioned media significantly increased EC, SMC, and C2C12 (an SkMB line) proliferation, by $39 \%, 41 \%$, and $27 \%$, respectively, compared with nonconditioned media, indicating that the secreted cytokines were functional (data not shown).

Effects of $m M A P C-U$ and $h M A P C-U$ in severe limb ischemia. Finally, we tested the potential of mMAPC-U, mMAPC-U2 (2 independently 

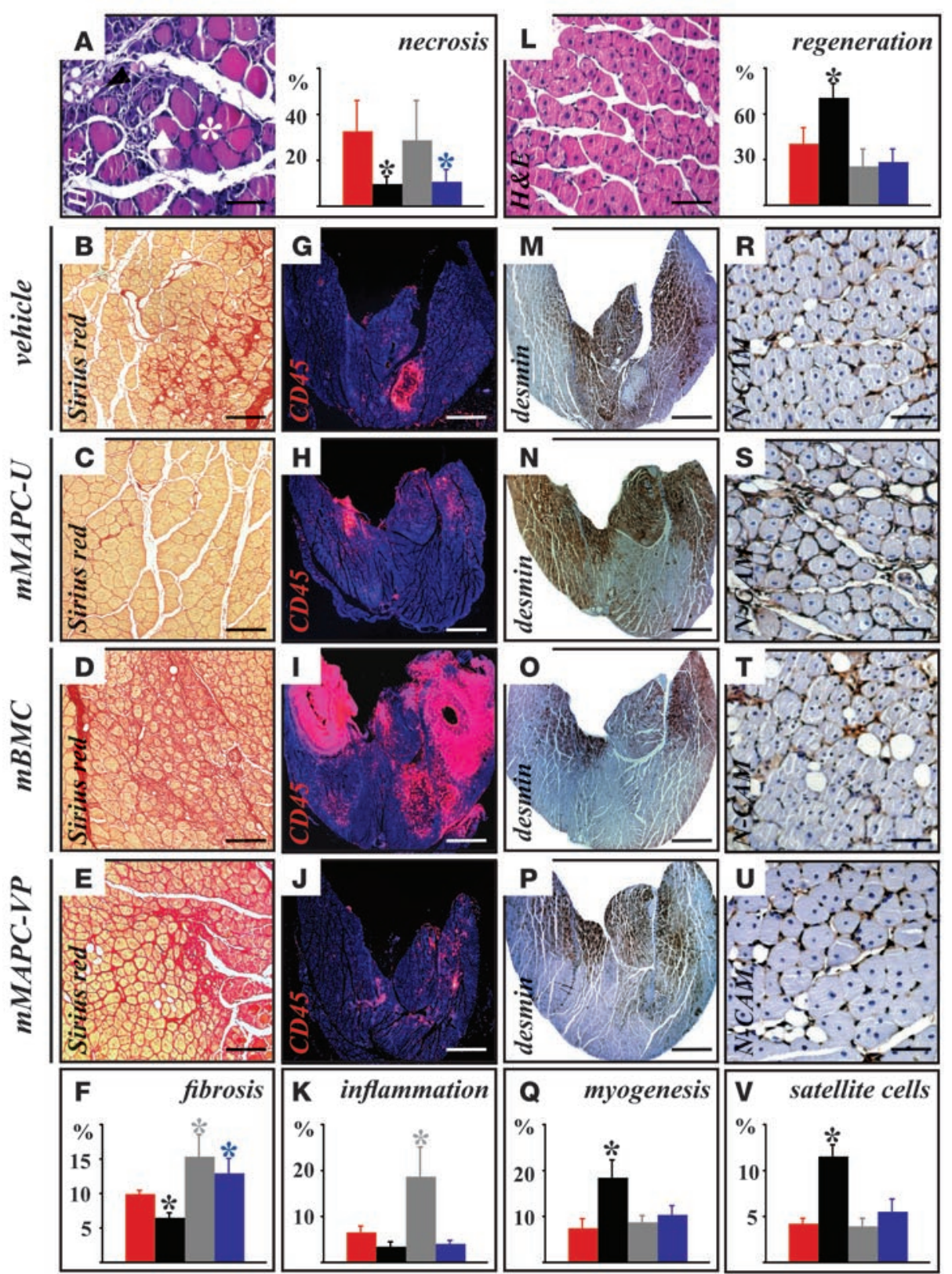



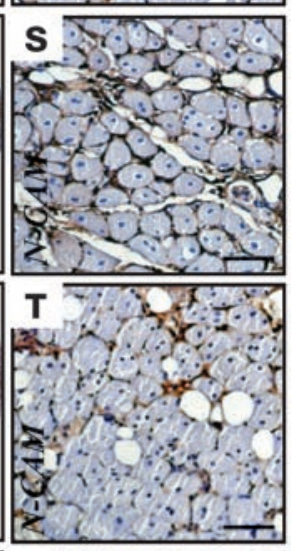

\section{Figure 5}

Differential effects of mMAPC-U, mMAPC-VP, and mBMCs in moderate ischemia. (A) Analysis of necrosis (defined as the presence of fat cells [black arrowhead], inflammatory infiltrates [white arrowhead], and "ghost" muscle cells devoid of a nucleus [asterisk]; expressed as \% versus total muscle area) on H\&E-stained cross sections in vehicle, mMAPC-U, mBMC, or mMAPC-VP groups. (B-F) Analysis of fibrosis on Sirius red-stained cross sections in vehicle (B), mMAPC-U (C), mBMC (D), or mMAPC-VP (E) groups. (G-K) Analysis of inflammation $\left(\mathrm{CD} 45^{+}\right.$area in red, expressed as \% versus total muscle area in $\mathbf{K}$ ) on cross sections in vehicle (G), mMAPC-U (H), mBMC (I), or mMAPC-VP (J) groups. (L) Analysis of muscle regeneration (fibers with central nuclei, expressed as \% versus total muscle area) on H\&E-stained cross sections in vehicle, mMAPC-U, mBMC, or mMAPC-VP groups. (M-Q) Analysis of myogenesis (desmin ${ }^{+}$area, expressed as $\%$ versus total muscle area in $\mathbf{Q}$ ) on cross sections in vehicle (M), mMAPC-U $(\mathbf{N}), \operatorname{mBMC}(\mathbf{O})$, or mMAPC-VP (P) groups. (R-V) Analysis of satellite cells $\left(\mathrm{N}-\mathrm{CAM}^{+}\right.$area, expressed as \% versus total muscle area in $\mathbf{V}$ ) on cross sections in vehicle (R), mMAPC-U (S), mBMC (T), or mMAPC-VP (U) groups. Red bars in $\mathbf{A}, \mathbf{F}, \mathbf{K}, \mathbf{L}, \mathbf{Q}$, and $\mathbf{V}$ correspond to vehicle, black bars to mMAPC-U, gray bars to $\mathrm{mBMC}$, and blue bars to MMAPC-VP groups. All images are from the gastrocnemius muscle. Hematoxylin was used as nuclear counterstain in $\mathbf{A}, \mathbf{L}, \mathbf{M}-\mathbf{P}$, and $\mathbf{R}-\mathbf{U}$ and DAPI in $\mathbf{G}-\mathbf{J}$. ${ }^{*} P<0.05$ versus vehicle. Scale bars: $62.5 \mu \mathrm{m}(\mathbf{A}$, $\mathbf{L}$, and $\mathbf{R}-\mathbf{U}), 130 \mu \mathrm{m}(\mathbf{B}-\mathbf{E})$, and $1 \mathrm{~mm}$ (G-J, M-P). derived clones), and hMAPC-U, transplanted 5 days after femoral or iliac ligation/transection in BALB/c-nu/nu mice, a model of severe limb ischemia, representative of CLI (25), as evidenced by the increased tissue necrosis rate (Table 1 versus Table 2 ). mMAPC-U, mMAPC-U2, and hMAPC-U grafting significantly expanded the collateral bed (2- to 3 -fold increase in $\alpha-\mathrm{SMA}^{+}$area), compared with control treatment (Figure 6, A-D and K, and Table 2 ) at 21 days. This was associated with a progressive improvement in perfusion (Figure 6E), significantly increased muscle viability (tetraphenyltetrazolium chloride [TTC] staining; Figure $6 \mathrm{~K}$ ), less fibrosis (Figure 6, G-K), and more muscle regeneration (Figure 6K) compared with control animals at 21 days (Table 2 ). Importantly, in all cell-transplanted groups, muscle function was restored more efficiently (Figure $6 \mathrm{~F}$ ) compared with that in the vehicle-injected group, and the incidence of toe necrosis/limb loss at 21 days was reduced 3 -fold (Table 2 ).

\section{Discussion}

Although it was hoped that revascularization by angiogenic growth factor therapy might be an alternative for surgical interventions in no-option PVD patients, results from clinical trials have been disappointing $(3,4)$. The other alternative, cell transplantation, might offer better perspectives, because it may overcome the problem of lack of response of endogenous vascular cells to growth factors and it may combine revascularization with replacement of lost tissue. Here, we tested these paradigms by delivery of exogenous vascular progenitors (mMAPC-VP) and by evaluating cell populations that have the ability to differentiate into vascular and muscle cells, namely mBMCs (18-21) and mMAPC-U $(22,23)$. We found that only mMAPC-U durably improved muscle function and perfusion due to the favorable combination of effects on revascularization and muscle regeneration, in both moderate and severe limb ischemia models. Furthermore, similar results were seen when hMAPCs were used in the severe model. 
Table 2

Effects of mMAPC-U and hMAPC-U in severe limb ischemia

\begin{tabular}{|c|c|c|c|c|}
\hline & Vehicle & mMAPC-U & mMAPC-U2 & hMAPC-U \\
\hline $\begin{array}{l}\text { Collaterals ( } \% \alpha \text {-SMA+ area); day } 21 ; n=6 \\
\text { Perfusional recovery }(\%) \cdot A \quad n=8-12\end{array}$ & $0.5 \pm 0.1$ & $1.5 \pm 0.2^{\mathrm{G}}$ & $1.2 \pm 0.1^{G}$ & $1.4 \pm 0.2^{G}$ \\
\hline Day 9 & $8 \pm 3$ & $17 \pm 3^{G}$ & $21 \pm 4^{G}$ & $14 \pm 3^{G}$ \\
\hline Day 14 & $9 \pm 3$ & $23 \pm 3^{G}$ & $25 \pm 5^{G}$ & $23 \pm 4^{G}$ \\
\hline Day 21 & $12 \pm 3$ & $23 \pm 3^{G}$ & $29 \pm 3^{G}$ & $25 \pm 4^{G}$ \\
\hline \multicolumn{5}{|l|}{ Treadmill endurance $(\%) ; B \quad n=7-9$} \\
\hline Day 9 & $163 \pm 16$ & $176 \pm 11$ & $151 \pm 12$ & $184 \pm 20$ \\
\hline Day 14 & $213 \pm 17$ & $270 \pm 24 \mathrm{H}$ & $243 \pm 22$ & $293 \pm 20 G$ \\
\hline Day 21 & $214 \pm 18$ & $345 \pm 43^{G}$ & $273 \pm 19$ & $336 \pm 21^{G}$ \\
\hline Tissue viability $(\%) ; c$ day $21 ; n=5-6$ & $31 \pm 8$ & $59 \pm 5^{G}$ & $56 \pm 7^{G}$ & $64 \pm 4^{G}$ \\
\hline Fibrosis $(\%) ;$ day $21 ; n=5-6$ & $29 \pm 2$ & $17 \pm 4^{G}$ & $14 \pm 3^{G}$ & $16 \pm 3^{G}$ \\
\hline Myogenesis $(\%) ;$ day $21 ; n=5-6$ & $12 \pm 1$ & $31 \pm 5^{G}$ & $18 \pm 3^{H}$ & $24 \pm 4^{G}$ \\
\hline Toe necrosis $(\%) ;$;ay $21 ; n=7-13$ & 91 & 29 & 31 & 33 \\
\hline
\end{tabular}

Data represent mean \pm SEM. A\% increase versus day 5 relative to nonligated leg; $\mathrm{B} \%$ of day 5 endurance; $\mathrm{C}_{\%} \mathrm{TTC}^{+}$area of total muscle area; $\mathrm{D} \%$ Sirius red ${ }^{+}$area of total muscle area; $\mathrm{E} \%$ desmin ${ }^{+}$area of total muscle area; Fincidence. $G P<0.05$ versus vehicle; ${ }^{H} P=0.07$ versus vehicle; ${ }^{I} P=0.05$ versus vehicle.

for mMAPC-U, and the improvement was no longer seen beyond 9 days after treatment. Moreover, we did not find durable contribution of mMAPC-VP to vessels. The differences between our results and those in the cranial window model may be due to the different tissue environments in the 2 models. Similar to mMAPC-VP and in line with other studies $(7,38,39)$, injection of $\mathrm{mBMCs}$ resulted in vascular bed expansion and improved perfusion and function of hind limb muscles; however, this too was temporary and less pronounced compared with mMAPC-U. Whether the lack of direct contribution of $\mathrm{mBMCs}$ to vessels and muscle tissue, or mMAPC-VP to skeletal muscle or their lower level of engraftment, underlies the significantly lower degree of improvement in perfusion or swim endurance compared

mMAPCs engrafted robustly up to 5 weeks, even though cell number decreased over time. As mMAPC expressed GFP - a well-known immunogen (30) - we believe that this decrease might be due to immune rejection by the host. Consistent with this is the presence of $\mathrm{CD}_{4} 5^{+}$cells surrounding implanted cells. The hypoxic $\left(5 \% \mathrm{O}_{2}\right)$ and low-serum $(2 \%)$ conditions under which mMAPCs are maintained (31) may have conditioned them to survive in ischemic environments. Such a favorable preconditioning effect was also documented for BMCs (32). Although mMAPCs only express low levels of CXCR4 (31), known to assist in perivascular positioning and retention (33), the grafted cells were consistently found in the immediate vicinity of vessels, a strategic position from which they could affect vascular bed expansion. Possibly, chemokine receptors and other adhesion receptors may be upregulated once mMAPCs are grafted in tissues.

mMAPCs differentiated into ECs and SMCs and contributed to SkMBs, consistent with their in vitro pluripotent differentiation properties $(22,23)$. While we did not observe fusion of mMAPCs with vascular cells, muscle contribution in vivo was at least in part due to fusion with host muscle cells; however, it remains to be determined whether the latter was via direct fusion with host myoblasts or differentiation to a muscle satellite stage and subsequent fusion (Supplemental Figure 2). In vitro, mMAPCs can differentiate into myotubes independent of fusion when cocultured with $\mathrm{C} 2 \mathrm{C} 12$ cells and also fuse with C2C12 cells (Supplemental Figure 2). As expected, vascular-committed mMAPCs did not contribute to skeletal muscle. Despite the presence of skeletal muscle precursors in BM (18), mBMCs also failed to differentiate in situ into SkMBs. This is in agreement with the observation that cells with high fusion capability mainly reside in the stromal fraction, while only a small fraction of the hematopoietic lineage is fusigenic (34). Other nonmuscle-derived cell types have shown the potential to contribute to SkMBs in ischemic muscle, e.g., CD $34^{+}$or $\mathrm{CD} 34^{+} \mathrm{KDR}^{+}$cord blood cells $(10,13)$, the immortalized CD34- $\mathrm{R} 26$ cell line generated from peripheral blood (35), or ADSCs (36).

A recent report has documented that cotransplantation of ECs with mesenchymal precursor cells in a cranial window model leads to the formation of durable, functional blood vessels (37). Although we demonstrate that mMAPC-VP induced an improvement in perfusion and function, this was less pronounced than with that of mMAPC-U remains to be determined. Also, what the contribution is of SkMB generation from mMAPC-U or the trophic support of endogenous myogenesis provided by mMAPC-U to the improved function is unclear. Nevertheless, muscle function of mMAPC-U-treated animals far exceeded that of the other study groups, in which no effects on muscle regeneration were seen.

It should be noted that the majority of mMAPC-U did not directly contribute to ECs, SMCs, or SkMBs. Although some earlier studies have suggested that grafted cells directly contribute to a much greater degree to vessels and/or tissue in ischemia models $(40,41)$, recent studies have demonstrated that direct contribution is minimal (9), or even absent $(11,42)$, and that grafted cells improve vascularization via trophic effects $(8,11)$. In our study, it is highly likely that trophic factors secreted by the long-term engrafted mMAPC-U, such as VEGF and IGF-1, contributed to the improved perfusion and limb function seen in mMAPC-U-treated animals.

A surprising finding was the progressive necrosis and fibrosis and the associated decrease in muscle function of mBMC-treated animals after day 9. It is noteworthy that aside from endogenous $\mathrm{CD} 45^{+}$cells, donor-derived immunological cells (or their progenitors) were also present in foci of inflammatory dendritic and $\mathrm{T}$ cells. In some animals, we saw an extensive infiltration of necrotic gastrocnemius muscle with $\mathrm{T}$ lymphocytes and dendritic cells, some of which were $\mathrm{GFP}^{+}$and hence donor in origin. Many preclinical studies have evaluated the benefits of $\mathrm{mBMC}$ transplantation in limb ischemia $(5,43,44)$; however, in most studies, cells were injected only in the upper part of the limb, where inflammation due to necrosis is limited, which may have precluded the detection of the contribution of grafted immune cells to the inflammatory reaction. While we used unfractionated BMCs, in most clinical studies, only the mononuclear fraction or subsets of these are used (Supplemental Table 1). Nevertheless, given the fact that these fractions still contain mature immune cells or precursors thereof, and that they are injected into the gastrocnemius muscle in the majority of PVD patient trials (Supplemental Table 1), our observation should serve as a note of caution, in that grafting of such cell populations in the ischemic, necrotic, and inflammatory limb muscle may contribute to and exacerbate the inflammatory response with potentially detrimental consequences later on. 



fibrosis



myogenesis



\section{Figure 6}

Effects of mMAPC-U and hMAPC-U in severe ischemia. (A-D) $\alpha$-SMA (red) staining at 21 days in adductor of vehicle- (A), mMAPC-U- (B), mMAPC-U2- (C), or hMAPC-U-treated mice (D). Overexposure in the DAPI channel was used to reveal muscle tissue. (E and $\mathbf{F}$ ) Laser Doppler measurements (E) in left legs (expressed as $\%$ improvement versus pretransplant [day +5 ] and relative to the nonligated right leg); and treadmill endurance test (F) measuring hind limb function (expressed as $\%$ of pretransplant performance [day +5$]$ ) at several time points after (+) ligation of vehicle- (red), mMAPC-U(black), mMAPC-U2- (gray), or hMAPC-U-treated mice (blue). (G-J) Sirius red-stained cross sections of gastrocnemius muscle 3 weeks after transplantation of vehicle- $(\mathbf{G})$, mMAPC-U- (H), mMAPC-U2- (I), or hMAPC-U-injected (J) animals. (K) Quantitative analysis of $\alpha$-SMA (in adductor), viability (TTC), fibrosis (Sirius red), and myogenesis (desmin) staining (all in gastrocnemius), expressed as percent positive staining per muscle area in vehicle- (red), mMAPC-U- (black), mMAPC-U2- (gray), or hMAPC-U-treated mice (blue). All analyses were performed on 6-12 mice per group. ${ }^{\star} P<0.05$ versus vehicle for each corresponding time point; ${ }^{\dagger} P=0.05$ versus vehicle; $\# P=0.07$ versus vehicle. Scale bars: $250 \mu \mathrm{m}(\mathbf{A}-\mathbf{D})$ and $62.5 \mu \mathrm{m}(\mathbf{G}-\mathbf{J})$.
Finally, we demonstrate that hMAPCs had a beneficial effect on limb perfusion and muscle regeneration similar to that of mMAPCs in a severe limb ischemia model. While these studies were done in immunocompromised mice, in a clinical setting, human recipients will be immunocompetent; if one is to consider allogeneic cells as graft, allogeneic hMAPCs, expressing HLA molecules upon differentiation, will likely also be rejected due to HLA incompatibility, as we have seen here for the neoantigen-containing GFP ${ }^{+}$ mMAPCs. Studies wherein mice with a "humanized" immune system are used will be needed to address the effect of HLA mismatch on the efficacy of hMAPCs in limb ischemia (45).

In summary, we show here that locally injected undifferentiated mMAPCs have the potential to remedy ischemic problems of the lower extremities in both moderate and severe ischemia models. Importantly, their human counterparts had a similar beneficial effect in the severe model. In the moderate model, the effect was superior to that of vascular-committed MAPCs or mBMCs, in both early and later phases. This greater potential may be related to the ability of the cells to stably engraft and induce vascular and skeletal muscle regeneration via direct contribution to vascular and skeletal muscle cells and via trophic effects on the endogenous vascular and skeletal muscle cells.

\section{Methods}

An extended Methods section is available as supplemental information.

Cell derivation and differentiation. Murine cells were derived from BMofC57BL/6 mice with ubiquitous GFP expression (mMAPC-U: C57BL/6-Tg-eGFP, a gift of I.L. Weissman, Stanford University, Stanford, California, USA;
mMAPC-U2: C57BL/6TgN (act-EGFP)ObsC14-YO1-FM1310, a gift of M. Okabe, Osaka University, Osaka, Japan). mMAPCs were derived and maintained under low $\mathrm{O}_{2}(5 \%)$ and low-serum $(2 \%)$ conditions and expressed the pluripotency transcription factor Oct-4 at levels between 5\% and 10\% of $\mathrm{mES}$ cells (31). GFP $\mathrm{GBMCs}^{+}$were isolated from the femurs, depleted of erythrocytes, washed, and transplanted. hMAPCs were established at the University of Navarra using BM from donors age 18-54, as described previously (46). Samples were obtained after informed consent was received from the donors according to guidelines of the Committee on the Use of Human Subjects in Research at the Clínica Universitaria, Pamplona. EC and SMC differentiation were performed as described previously $(23,24)$.

Mice, surgery, and transplantation. For the moderate limb ischemia model, recipient mice were C57BL/6 males (age 12-16 weeks). Ischemia was induced under anesthesia (100 mg/kg ketamine plus $10 \mathrm{mg} / \mathrm{kg}$ xylocaine) by ligation of the left and, where indicated, right common femoral arteries. Cells $\left(1 \times 10^{6}\right.$ in total), resuspended in PBS, or PBS alone were injected in equal fractions in the adductor and lower calf region (Supplemental Figure 1 , A and B), always on the left side, immediately after ligation.

For the severe limb ischemia model, recipient mice were BALB/c-nu/nu males (age 12-16 weeks). Ischemia was induced under anesthesia $(100 \mathrm{mg} / \mathrm{kg}$ ketamine plus $10 \mathrm{mg} / \mathrm{kg}$ xylocaine) by ligation/transection of the left iliac artery (laser Doppler studies) or bilateral ligation/transection of the common femoral arteries (treadmill studies). Cells $\left(1 \times 10^{6}\right.$ in total), resuspended in PBS, or PBS alone were injected as described above (Supplemental Figure 1, A and B); however, cells were injected 5 days after surgery, and for treadmill studies, both limbs were transplanted.

As MAPC-U do not express MHC-I and, consequently, are sensitive to NK cell-mediated clearance, all mice were injected i.p. with $20 \mu \mathrm{l}$ anti-asialo GM1 
antibodies (20x diluted in PBS; Wako Chemicals) 1-2 hours before transplantation and every 10 days thereafter. These antibodies selectively eliminate NK cells without affecting the macrophage or lymphocyte function (47). Mice were housed in specific pathogen-free conditions, and procedures involving animals were approved by the Institutional Animal Care and Use Committees of the Universities of Minnesota, Navarra, and Leuven.

Live imaging and assessment of limb function and blood flow/perfusion. Live imaging was performed using a Leica dissection microscope after anesthetizing the mice with $60 \mathrm{mg} / \mathrm{kg}$ Nembutal and gently removing the skin from the hind limbs. Limb function was evaluated by a swim endurance test, as described previously (26) (moderate model), or a treadmill test (severe model). Muscle energetic status was determined, under temperature-controlled conditions, by MRSI, using a 9.4 Tesla horizontal magnet (Magnex Scientific) interfaced with a Unity INOVA console (Varian Inc.). Blood flow was quantified by laser Doppler probing at anatomically fixed locations from below knee level to foot (moderate model; Moor Instruments) or by whole-limb scanning (severe model; Lisca PIM II) under temperature-controlled conditions, as described previously (26).

Tissue processing, histology, and microscopy. At distinct time points after ligation, mice were anesthetized with $60 \mathrm{mg} / \mathrm{kg}$ Nembutal and perfused with trisbuffered saline-adenosine for 2 minutes followed by zinc paraformaldehyde for 18 minutes. Following dissection, muscles were fixed for an additional 24 hours, divided in 2 equal pieces, and processed for paraffin or OCT embedding. For all stainings and analyses, $7-\mu \mathrm{m}$ sections were used, spanning a muscle area of approximately $2 \mathrm{~mm}$ in thickness. H\&E and Sirius red stainings were performed as described previously (46). For immunohistochemistry and immunofluorescence, a list of primary antibodies is provided as supplemental information (Supplemental Table 2). Whole muscle viability assays were performed on unfixed tissue slices of approximately $2 \mathrm{~mm}$ in thickness after staining with TTC, as described previously (48). Pictures for morphometric analysis were taken using a Retiga EXi camera (Q Imaging) connected to a Nikon E800 microscope or a Zeiss Axio Imager connected to an Axiocam MRc5 camera (Zeiss), and analysis was performed using ImageJ (http://rsb. info.nih.gov/ij/), KS300 (Leica), or Openlab 3.1 (Improvision) software. Confocal microscopy was performed on a Zeiss LSM 510.

RNA isolation, quantitative RT-PCR, cytokine production, cell proliferation/ differentiation assays. Total RNA isolation, cDNA synthesis, and quantitative RT-PCR were performed as described in Supplemental Methods. Primers used for amplification are provided in Supplemental Table 3. To assess cytokine production of mMAPC-U, cells were plated in triplicate in cytokine-free and low-serum expansion media, and supernatant was collected 60 hours later and frozen. mBMCs were plated in DMEM supplemented with $10 \%$ serum. ELISA kits were from R\&D Systems, and the procedure was performed according to the manufacturer's recommendations. For proliferation assays, MS-I ECs, C2C12 myoblasts (both from ATCC), and RaoSMCs (Cell Applications) were plated in triplicate; 2 hours later, $75 \%$ of the media was replaced with conditioned media in half of the wells, and in the other half, $75 \%$ of the media was replaced

1. Collinson, D.J., and Donnelly, R. 2004. Therapeutic angiogenesis in peripheral arterial disease: can biotechnology produce an effective collateral circulation? Eur. J. Vasc. Endovasc. Surg. 28:9-23.

2. Norgren, L., et al. 2007. Inter-society consensus for the management of peripheral arterial disease (TASC II). J. Vasc. Surg. 45(Suppl. S):S5-S67.

3. Makinen, K., et al. 2002. Increased vascularity detected by digital subtraction angiography after VEGF gene transfer to human lower limb artery: a randomized, placebo-controlled, double-blinded phase II study. Mol. Ther. 6:127-133.

4. Rajagopalan, S., et al. 2003. Regional angiogenesis with vascular endothelial growth factor in peripheral arterial disease: a phase II randomized, double-

with unconditioned media that had been incubated for 60 hours in the absence of cells. In order to avoid artifacts due to serum depletion during the 60-hour incubation, serum levels were replenished. Seventy-two hours later, cells were harvested by trypsinization and counted. To study fusion in vitro, MAPC-U were cocultured with PKH26-labeled C2C12 cells under proliferating conditions. From day 4 to 10, 50\% of the media was replaced with serum-free media to induce differentiation. Cultures were screened by confocal microscopy.

Statistics. For comparisons of 2 groups, data, expressed as mean \pm SEM, were analyzed using the 2-tailed unpaired Student's $t$ test. Comparisons among 4 groups were analyzed by ANOVA. A Kolmogorov-Smirnov test was used to verify that the data had a Gaussian distribution, which justified the use of a parametric test. Instat3.0a software (GraphPad Software Inc.) was used for statistical analyses, and differences were considered statistically significant when $P$ was less than 0.05 .

\section{Acknowledgments}

The authors thank A. Breyer, L. Lien, N. Estharabadi, E. Lee, D. Anderson, and J. Browers (University of Minnesota), M. Ecay and M. Collantes (CIMA, Pamplona), and P. Vandervoort and Tom Vervoort (University of Leuven) for excellent technical assistance; as well as W. Low (University of Minnesota) for vacating the laser Doppler equipment and Y. Nakagawa (University of Minnesota) for vacating the Nikon E800 microscope and Openlab 3.1 software. This work was supported by grants from the Belgian American Educational Foundation (BAEF; to A. Luttun), the American Heart Association (to A. Luttun and B. Pelacho), the European Commission (StrokeMAP STREP-FP6; to X.L. Aranguren, A. Luttun, F. Prósper, and C.M. Verfaillie), the Spanish government (SAF 2002-04574-C02 and PI050168; to F. Prósper), FEDER (Interreg IIIA; to F. Prósper), UTE project CIMA (to F. Prósper), an FWO postdoctoral fellowship (to A. Luttun), an FWO predoctoral fellowship (to B. Hendrickx), an FWO Odysseus award (to C.M. Verfaillie), 2 KU Leuven Center of Excellence grants (A. Luttun, EF/05/013, and C.M. Verfaillie), R01 DK-58295 from the NHLBI (C.M. Verfaillie) and sponsored research project (A10) from Athersys Inc. (C.M. Verfaillie).

Received for publication December 6, 2006, and accepted in revised form October 22, 2007.

Address correspondence to: Aernout Luttun, Center for Molecular and Vascular Biology, Katholieke Universiteit Leuven, Campus Gasthuisberg, Herestraat 49, B-3000 Leuven, Belgium. Phone: 32-16-34-57-72; Fax: 32-16-34-59-90; E-mail: aernout.luttun@ med.kuleuven.be.

Jonathan D. McCue and Benoit Hendrickx contributed equally to this work.

blind, controlled study of adenoviral delivery of vascular endothelial growth factor 121 in patients with disabling intermittent claudication. Circulation. 108:1933-1938.

5. Jeon, O., et al. 2006. Synergistic effect of sustained delivery of basic fibroblast growth factor and bone marrow mononuclear cell transplantation on angiogenesis in mouse ischemic limbs. Biomaterials. 27:1617-1625

6. Asahara, T., et al. 1997. Isolation of putative progenitor endothelial cells for angiogenesis. Science. 275:964-967.

7. Ikenaga, S., et al. 2001. Autologous bone marrow implantation induced angiogenesis and improved deteriorated exercise capacity in a rat ischemic hindlimb model. J. Surg. Res. 96:277-283.

8. Kinnaird, T., et al. 2004. Local delivery of marrowderived stromal cells augments collateral perfusion through paracrine mechanisms. Circulation. 109:1543-1549.

9. Kupatt, C., et al. 2005. Embryonic endothelial progenitor cells expressing a broad range of proangiogenic and remodeling factors enhance vascularization and tissue recovery in acute and chronic ischemia. FASEB J. 19:1576-1578.

10. Madeddu, P., et al. 2004. Transplantation of low dose CD34+KDR+ cells promotes vascular and muscular regeneration in ischemic limbs. FASEB J. 18:1737-1739.

11. Nakagami, H., et al. 2005. Novel autologous cell 
therapy in ischemic limb disease through growth factor secretion by cultured adipose tissuederived stromal cells. Arterioscler. Thromb. Vasc. Biol. 25:2542-2547.

12. Niagara, M.I., et al. 2004. Autologous skeletal myoblasts transduced with a new adenoviral bicistronic vector for treatment of hind limb ischemia. J. Vasc. Surg. 40:774-785.

13. Pesce, M., et al. 2003. Myoendothelial differentiation of human umbilical cord blood-derived stem cells in ischemic limb tissues. Circ. Res. 93:e51-e62.

14. Dimmeler, S., Zeiher, A.M., and Schneider, M.D. 2005. Unchain my heart: the scientific foundations of cardiac repair. J. Clin. Invest. 115:572-583.

15. Emmerich, J. 2005. Current state and perspective on medical treatment of critical leg ischemia: gene and cell therapy. Int. J. Low. Extrem. Wounds. 4:234-241.

16. Heng, B.C., Cao, T., Haider, H.K., Rufaihah, A.J., and Sim, E.K. 2005. Utilizing stem cells for myocardial repair--to differentiate or not to differentiate prior to transplantation? Scand. Cardiovasc. J. 39:131-134.

17. Urbich, C., et al. 2005. Cathepsin $\mathrm{L}$ is required for endothelial progenitor cell-induced neovascularization. Nat. Med. 11:206-213.

18. Torrente, Y., et al. 2004. Human circulating AC133 $(+)$ stem cells restore dystrophin expression and ameliorate function in dystrophic skeletal muscle. J. Clin. Invest. 114:182-195.

19. Lamagna, C., and Bergers, G. 2006. The bone marrow constitutes a reservoir of pericyte progenitors. J. Lenkoc. Biol. 80:677-681.

20. Shimizu, K., et al. 2001. Host bone-marrow cells are a source of donor intimal smooth- muscle-like cells in murine aortic transplant arteriopathy. Nat. Med. 7:738-741.

21. Urbich, C., and Dimmeler, S. 2004. Endothelial progenitor cells: characterization and role in vascular biology. Circ. Res. 95:343-353.

22. Jiang, Y., et al. 2002. Pluripotency of mesenchymal stem cells derived from adult marrow. Nature. 418:41-49.

23. Reyes, M., et al. 2002. Origin of endothelial progenitors in human postnatal bone marrow. J. Clin. Invest. 109:337-346.

24. Ross, J.J., et al. 2006. Cytokine-induced differentiation of multipotent adult progenitor cells into functional smooth muscle cells. J. Clin. Invest. 116:3139-3149.

25. Waters, R.E., Terjung, R.L., Peters, K.G., and Annex, B.H. 2004. Preclinical models of human peripheral arterial occlusive disease: implications for investigation of therapeutic agents. J. Appl. Physiol. 97:773-780.

26. Luttun, A., et al. 2002. Revascularization of ischemic tissues by PlGF treatment, and inhibition of tumor angiogenesis, arthritis and atherosclerosis by anti-Flt1. Nat. Med. 8:831-840.

27. Shim, J.Y., and Kim, T.S. 2003. Relationship between utrophin and regenerating muscle fibers in Duchenne muscular dystrophy. Yonsei Med. J. 44:15-23.

28. Salimena, M.C., Lagrota-Candido, J., and QuiricoSantos, T. 2004. Gender dimorphism influences extracellular matrix expression and regeneration of muscular tissue in mdx dystrophic mice. Histochem. Cell Biol. 122:435-444.

29. Heil, M., and Schaper, W. 2004. Influence of mechanical, cellular, and molecular factors on collateral artery growth (arteriogenesis). Circ. Res. 95:449-458.

30. Andersson, G., et al. 2003. Engraftment of retroviral EGFP-transduced bone marrow in mice prevents rejection of EGFP-transgenic skin grafts. $\mathrm{Mol}$. Ther. 8:385-391.

31. Ulloa-Montoya, F., et al. 2007. Comparative transcriptome analysis of embryonic and adult stem cells with extended and limited differentiation capacity. Genome Biol. 8:R163.

32. Li, T.S., et al. 2002. Improved angiogenic potency by implantation of ex vivo hypoxia prestimulated bone marrow cells in rats. Am. J. Physiol. Heart Circ. Physiol. 283:H468-H473.

33. Grunewald, M., et al. 2006. VEGF-induced adult neovascularization: recruitment, retention, and role of accessory cells. Cell. 124:175-189.

34. Shi, D., Reinecke, H., Murry, C.E., and Torok-Storb, B. 2004. Myogenic fusion of human bone marrow stromal cells, but not hematopoietic cells. Blood. 104:290-294.

35. Huss, R., et al. 2004. Improved arteriogenesis with simultaneous skeletal muscle repair in ischemic tissue by SCL $(+)$ multipotent adult progenitor cell clones from peripheral blood. J. Vasc. Res. 41:422-431.

36. Di Rocco, G., et al. 2006. Myogenic potential of adi- pose-tissue-derived cells. J. Cell Sci. 119:2945-2952.

37. Koike, N., et al. 2004. Tissue engineering: creation of long-lasting blood vessels. Nature. 428:138-139.

38. Finney, M.R., et al. 2006. Direct comparison of umbilical cord blood versus bone marrow-derived endothelial precursor cells in mediating neovascularization in response to vascular ischemia. Biol. Blood Marrow Transplant. 12:585-593.

39. Tateno, K., et al. 2006. Critical roles of musclesecreted angiogenic factors in therapeutic neovascularization. Circ. Res. 98:1194-1202.

40. Kalka, C., et al. 2000. Transplantation of ex vivo expanded endothelial progenitor cells for therapeutic neovascularization. Proc. Natl. Acad. Sci. U. S. A. 97:3422-3427.

41. Kocher, A.A., et al. 2001. Neovascularization of ischemic myocardium by human bone-marrowderived angioblasts prevents cardiomyocyte apoptosis, reduces remodeling and improves cardiac function. Nat. Med. 7:430-436.

42. Zentilin, L., et al. 2006. Bone marrow mononuclear cells are recruited to the sites of VEGF-induced neovascularization but are not incorporated into the newly formed vessels. Blood. 107:3546-3554.

43. Hamano, K., et al. 2001. The induction of angiogenesis by the implantation of autologous bone marrow cells: a novel and simple therapeutic method. Surgery. 130:44-54.

44. Shintani, S., et al. 2001. Augmentation of postnatal neovascularization with autologous bone marrow transplantation. Circulation. 103:897-903.

45. Traggiai, E., et al. 2004. Development of a human adaptive immune system in cord blood cell-transplanted mice. Science. 304:104-107.

46. Aranguren, X.L., et al. 2007. In vitro and in vivo arterial differentiation of human multipotent adult progenitor cells. Blood. 109:2634-2642.

47. Seaman, W.E., Sleisenger, M., Eriksson, E., and Koo, G.C. 1987. Depletion of natural killer cells in mice by monoclonal antibody to NK-1.1. Reduction in host defense against malignancy without loss of cellular or humoral immunity. J. Immunol. 138:4539-4544.

48. Ytrehus, K., et al. 1994. Rat and rabbit heart infarction: effects of anesthesia, perfusate, risk zone, and method of infarct sizing. Am. J. Physiol. 267:H2383-H2390. 